\title{
Home Sleep Tests for Obstructive Sleep Apnea (OSA)
}

\author{
Mukesh Kapoor, MD, and Glen Greenough, MD
}

Obstructive sleep apnea (OSA) is a fairly common condition that, if left untreated, can lead to complications such as high blood pressure and heart disease. Polysomnography (PSG) is the most accurate method for diagnosing OSA, but it is a cumbersome and expensive test. A well-validated, easier to perform and less expensive alternative is the home sleep test (HST). The purpose of this review is to educate the primary care provider about the important differences between PSG and HSTs, the advantages and limitations of both modalities, identifying patients who are appropriate candidates for the HST, identifying patients in whom the HST should not be performed, and further evaluation of patients who have a negative HST. (J Am Board Fam Med 2015;28:504-509.)

Keywords: Home Sleep Testing, Monitoring, Obstructive Sleep Apnea, Out of Center Sleep Testing, Portable Monitoring

Obstructive sleep apnea (OSA) is thought to affect between $3 \%$ and $7 \%$ of the population, ${ }^{1}$ although a significantly higher prevalence is seen in certain subgroups of patients, such as those with atrial fibrillation, ${ }^{2,3}$ congestive heart failure, ${ }^{4,5}$ resistant hypertension, ${ }^{6}$ type 1 diabetes mellitus, ${ }^{7}$ type 2 diabetes mellitus, ${ }^{8}$ and morbid obesity. ${ }^{9}$ One study showed that approximately $32 \%$ of patients in a primary care setting have a high pretest probability for OSA. ${ }^{10}$ Some symptoms associated with OSA include snoring, observed pauses in breathing during sleep, frequent awakenings, nocturia, nonrefreshing sleep, and feeling tired and sleepy during the day. Untreated OSA can increase the risk for developing other medical problems, including high blood pressure and heart disease. ${ }^{11}$

The most accurate method for diagnosing OSA is by polysomnography (PSG), which requires that the patient sleep overnight in a sleep laboratory. Multiple sensors are used during

This article was externally peer reviewed.

Submitted 8 September 2014; revised 8 February 2015; accepted 23 February 2015.

From the Sleep Disorders Center, Dartmouth Hitchcock Medical Center, Lebanon, NH.

Funding: none.

Conflict of interest: none declared.

Corresponding author: Mukesh Kapoor, MD, 18 Old Etna Road, Lebanon, NH 03766 (E-mail: mukeshkapoor2011@ u.northwestern.edu).
PSG, including electroencephalography (EEG) leads, which record brain activity; electrooculography leads, which measure eye movement; electromyography leads, which measure muscle tone; a nasal pressure transducer and oronasal thermistor, which measures respiratory airflow; respiratory inductance plethysmography belts, which measure respiratory effort; electrocardiography leads, which measure cardiac activity; a pulse oximeter probe, which measures oxygen saturation; and limb leads, which measure limb movements. EEG allows for the detection of different sleep stages, analysis of sleep architecture, differentiation between awake and sleep, and identification of abnormal brain activity such as seizures. Electrooculography and electromyography help in the detection of rapid eye movement (REM) sleep, as there are characteristic eye movements and loss of muscle tone during this sleep stage. Trained sleep technologists set up the patient for PSG in the sleep laboratory and use a video camera to monitor the patient during this test. The position of the patient (supine vs. side vs. prone) and abnormal body movements can be visually recorded. Some patients can have positional sleep apnea, which is more severe in the supine position and less so when positioned on the side. ${ }^{12}$ If a patient is spending the majority of the night on their side, the technologist can intervene and have them spend some time on their back to look for such 
a positional change. ${ }^{13}$ If there is evidence of significant OSA during the early part of this test, the technologist can initiate continuous positive airway pressure titration and continue this for the remainder of the study time.

Some patients undergoing PSG may experience anxiety and discomfort associated with sleeping outside of the home environment. Depending on the geographic location, PSG might be unavailable or there might be a long wait time before a patient can undergo this test. ${ }^{14,15}$ PSG is cumbersome because multiple sensors (detailed above) are used, and it can be uncomfortable for some patients. PSG is also expensive; reimbursement from the Centers for Medicare \& Medicaid Services (CMS) is approximately $\$ 700$.

A well-validated alternative to PSG is the home sleep test (HST). ${ }^{16-22}$ This is also referred to as a portable monitor or an out-of-center sleep test. As the name of this test suggests, the patient stays in their home when it is performed. ${ }^{13}$ This is especially beneficial for patients who might feel anxious staying overnight in a sleep laboratory for PSG. The HST has fewer sensors, is more compact, and is less cumbersome to perform. A technologist is not needed to monitor the patient during the HST. Given the prevalence of OSA, as discussed earlier, the HST can be a useful tool for primary care providers to evaluate their patients suspected to have this disorder. The HST can aid in the early diagnosis and initiation of treatment in these patients. The HST is also less expensive; reimbursement from the CMS is approximately $\$ 250$.

The HSTs most commonly used in routine clinical practice have sensors that measure only respiratory airflow, respiratory effort, and oxygen saturation. Some HSTs have a built-in sensor to detect body position and an actigraph, which can be used as a surrogate marker for identifying when the patient is awake and sleep. ${ }^{13}$ The majority of commonly used HSTs do not include EEG monitoring. The few HSTs that do have the capability of EEG monitoring are not widely available and are more cumbersome and expensive.

Patients with OSA can have several different kinds of respiratory abnormalities. They can have apneas, which are defined as the cessation of breathing for $\geq 10$ seconds. Another type of respiratory abnormality is the hypopnea, which represents attenuation in the depth of respiration for at least 10 seconds that leads to desaturation or arousal from sleep. Patients may also have other respiratory abnormalities during which there is increased effort to breathe, leading to an arousal. These episodes are called respiratory effort-related arousals. The above respiratory abnormalities have very specific technical definitions, and the reader is referred to the American Academy of Sleep Medicine (AASM) guidelines on scoring respiratory events for further details. ${ }^{23}$

The principal way to detect arousals from sleep is through the use of EEG. It is important for health care providers to recognize that the most commonly used HSTs do not typically include EEG and thus cannot detect respiratory events that lead only to an arousal. Thus a patient with OSA consisting of predominantly arousal-based respiratory events can have a negative HST. This is especially important in pediatric patients and patients who have a low body mass index (BMI) because both are more likely to have respiratory events that lead to an arousal rather than desaturation. ${ }^{24,25}$

The parameters which are commonly measured by a HST are also recorded during PSG in addition to several other variables including brain activity through EEG. Thus, having EEG during PSG allows for the detection of respiratory events that are associated with arousals. The EEG component of PSG also allows one to know when a patient is awake and when they are asleep, providing a more accurate assessment for the presence of OSA and its severity. For example, a patient undergoing PSG may spend 10 hours in bed on the night of the test, but the EEG may show that the patient actually slept for only 5 hours. If a total of 100 respiratory events were recorded during the test, one would calculate the severity of this patient's sleep apnea at 100 events in 5 hours, or 20 events/h. By contrast, a HST cannot differentiate between wake and sleep. If the above patient were to undergo a HST, the severity of their sleep apnea would be calculated at 100 events (in all likelihood the HST would detect fewer of the respiratory events as well, but for argument's sake we will leave the number at $100)$ in the total recording time of 10 hours, or 10 events/h. Thus a HST tends to underestimate the severity of sleep apnea. This is especially of consequence in patients with mild sleep apnea in whom the HST can be negative because of the above. While some HSTs use actigraphy to differentiate between wake and sleep, one must remember that this is only a surrogate marker and can give flawed 
results, particularly in the presence of periodic limb movements, body movements following an arousal, and in patients with REM sleep behavior disorder. ${ }^{13}$

The AASM recommends that the HST should be used only in patients with a high pretest probability for moderate to severe OSA. ${ }^{26}$ Unfortunately, in our current health care environment, the majority of patients suspected of having OSA are channeled toward getting the HST, irrespective of their pretest probability. ${ }^{27}$ This increases the risk of having false-negative results with the HST. Many patients and clinicians are falsely reassured by a nondiagnostic HST. Not acting on these negative results by pursuing PSG can leave a patient with OSA undiagnosed.

A study published by a referral sleep disorders center looked at 187 patients who were suspected of having OSA (history of snoring along with an Epworth score $>7$ and/or a history of tiredness) but had a negative HST. ${ }^{28}$ All these patients underwent PSG, which showed that $90 \%$ of them had OSA $(26 \%$ had mild OSA, $43 \%$ had moderate OSA, and $21 \%$ had severe OSA). The fact that such a significant number of patients with a negative HST were found to be positive for OSA on PSG was likely secondary to several factors, including the inability of the HST device to detect respiratory events which were only associated with arousals, the use of total recording time in lieu of total sleep time, and the use of the HST device in a population of patients who might not have had a high pretest probability for moderate to severe OSA.

The AASM recommends that the HST should not be used in patients with significant comorbid medical conditions (such as those with moderate to severe pulmonary disease, neuromuscular disease, and congestive heart failure) that may degrade the accuracy of this test and in patients suspected of having other sleep disorders (such as central sleep apnea, periodic limb movement disorder, insomnia, parasomnias, circadian rhythm disorders, narcolepsy, and REM sleep behavior disorder). ${ }^{26}$ The AASM also recommends against using the HST for screening asymptomatic populations. ${ }^{26}$

There are no clear-cut guidelines for sleep testing in obese (BMI $\geq 30 \mathrm{~kg} / \mathrm{m}^{2}$ ) patients suspected of having OSA. Obesity by itself is not an indication for obtaining a PSG. Obese patients who are suspected of having obesity hypoventilation syndrome (OHS), however, should undergo PSG. The incidence of $\mathrm{OHS}$ increases with an increase in
BMI. ${ }^{29-31}$ It is therefore advisable to perform PSG in patients with morbid obesity (BMI $\geq 40 \mathrm{~kg} / \mathrm{m}^{2}$ ). Primary care physicians frequently have patients with hypertension and diabetes mellitus. Neither of these conditions is a contraindication to performing the HST.

As mentioned earlier, the most commonly used HSTs lack EEG monitoring and thus should not be used in patient suspected of having nocturnal seizures. Other patients who are poor candidates for the HST include those in whom hypoventilation (such as patients using narcotics, those who have neuromuscular disease, or those who have a high $\mathrm{BMI}$ and could have OHS) or nocturnal arrhythmias are suspected. ${ }^{32}$ Patients who have low oxygen saturation at baseline or are receiving supplemental oxygen are also poor candidates for the HST. ${ }^{32}$ In addition, the HST should not be used in pediatric patients because it can miss arousal-based hypopneas, which tend to be more frequent in this population. ${ }^{24}$

If the patient spends the majority of the night on their side or outside of REM sleep, one might never know the true severity of their OSA because the latter tends to be worse in the supine position and during REM sleep. ${ }^{12}$ Some HSTs have a body position monitor whereas others do not. The most commonly available HSTs do not have EEG monitoring and therefore cannot record sleep stages. Body position and sleep stages are routinely recorded during PSG. This can quantify the exact duration of time spent by the patient in the supine position and in REM sleep. Other problems with the HST include a data loss rate of between $3 \%$ and $18 \% .{ }^{13}$ As opposed to PSG, loss of a sensor signal cannot be corrected during a HST because of the lack of live monitoring and the absence of a technologist during this test. For similar reasons, continuous positive airway pressure titration cannot be performed during a HST. ${ }^{13}$

There is a unique HST that collects peripheral arterial tone (PAT), oxygen saturation, heart rate, and actigraphy data. ${ }^{32}$ This device is validated for the diagnosis of OSA. ${ }^{22,33-36}$ The PAT signal is a measure of sympathetic activity and is augmented, along with an increase in the heart rate and a decrease in oxygen saturation, at the termination of a respiratory event. ${ }^{32,34}$ Using the above measurements, this device can indirectly detect respiratory events. It also has an built-in algorithm that uses the PAT and actigraphy data to estimate sleep stages. ${ }^{32}$ This device does not, however, measure direct respiratory flow and respira- 
tory effort and thus cannot distinguish between central apneas, obstructive apneas, and hypopneas. ${ }^{34}$ In addition, this device has no way of quantifying the cause of increased sympathetic activity which can also occur in patients who have periodic limb movements. ${ }^{34}$ Lastly, the detection of sleep stages by this device has only moderate agreement with the more standard technique of PSG. ${ }^{37}$

Figure 1. A suggested algorithm for using home sleep testing (HST) in adult patients suspected of having obstructive sleep apnea (OSA). Note that HST should not be used in pediatric patients. *Significant comorbid medical disorders include conditions such as moderate to severe pulmonary disease, neuromuscular disease, and congestive heart failure which may degrade the accuracy of the home sleep test. Patients who have low baseline oxygen saturation or are on supplemental oxygen are also poor candidates for a home sleep test. Note that hypertension and diabetes mellitus are not contraindications for obtaining a home sleep test. See manuscript text for discussion on decision making in obese patients. †Comorbid sleep disorders include conditions such as central sleep apnea, periodic limb movement disorder, insomnia, parasomnias, circadian rhythm disorders, narcolepsy, and REM sleep behavior disorder. Central sleep apnea is often seen in patients with heart failure and those on narcotic medications. $\$$ Causes of hypoventilation include neuromuscular disease, restrictive lung disease, and use of narcotic medications. Patients with an elevated body mass index are also at risk for hypoventilation. PSG, polysomnography. (Adapted with permission from Collop et al. ${ }^{26}$ )

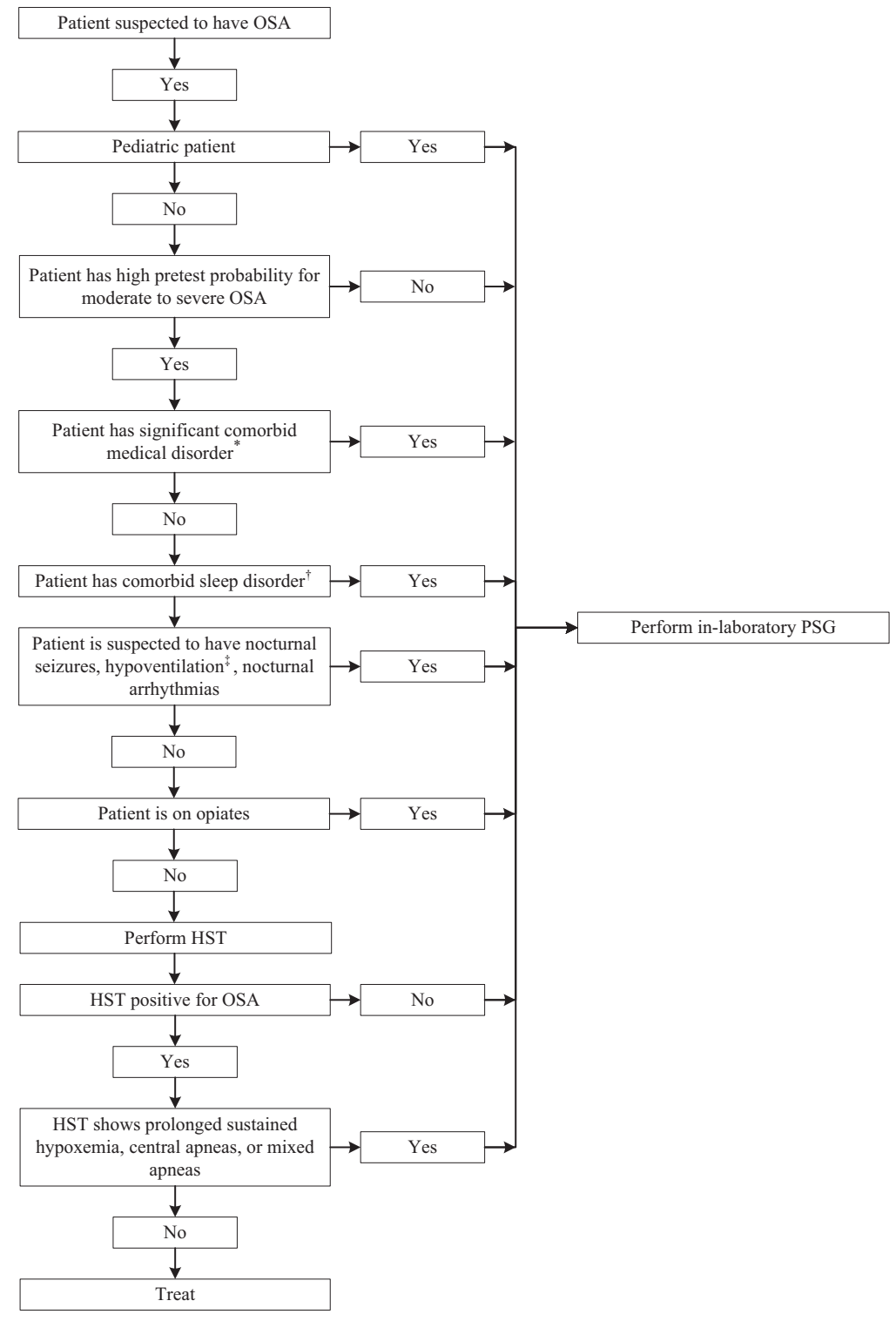


When faced with a patient suspected of having OSA, a primary care provider may choose either to refer their patient to a board-certified sleep specialist for further evaluation or to directly order a HST. When electing to do the latter, the primary care provider should be aware of the type of device being used, along with its sensitivity and specificity in different populations. The presence of prolonged sustained hypoxemia, central apneas, mixed apneas, or a negative HST are indications for referring a patient for PSG. Other things to keep in mind are that at least 300 minutes of recording time are needed during a HST for accurate diagnosis of OSA and its severity, ${ }^{38}$ and that manual scoring is superior to automatic scoring. ${ }^{39-42}$ A suggested algorithm for using the HST is shown in Figure 1.

The HST is an important tool if used and interpreted in the right manner in the right population. A patient's symptoms and examination must be kept in mind when determining the appropriateness for ordering a HST, which has the potential to save money, is easy to perform, improves access to sleep testing, and can be done in the patient's home. It must be remembered, however, that a patient suspected of having OSA and a negative HST should undergo PSG for further diagnostic evaluation. ${ }^{26,28}$

\section{References}

1. Punjabi NM. The epidemiology of adult obstructive sleep apnea. Proc Am Thorac Soc 2008;5:136-43.

2. Gami AS, Pressman G, Caples SM, et al. Association of atrial fibrillation and obstructive sleep apnea. Circulation 2004;110:364-7.

3. Patel D, Mohanty P, Di Biase L, et al. Safety and efficacy of pulmonary vein antral isolation in patients with obstructive sleep apnea: the impact of continuous positive airway pressure. Circ Arrhythm Electrophysiol 2010;3:445-51.

4. Javaheri S. Sleep disorders in systolic heart failure: a prospective study of 100 male patients. The final report. Int J Cardiol 2006;106:21-8.

5. Yumino D, Wang H, Floras JS, et al. Prevalence and physiological predictors of sleep apnea in patients with heart failure and systolic dysfunction. J Card Fail 2009; 15:279-85.

6. Florczak E, Prejbisz A, Szwench-Pietrasz E, et al. Clinical characteristics of patients with resistant hypertension: the RESIST-POL study. J Hum Hypertens 2013;27:678-85.

7. Manin G, Pons A, Baltzinger P, et al. Obstructive sleep apnoea in people with Type 1 diabetes: prevalence and association with micro- and macrovascular complications. Diabet Med 2014;32:90-6.
8. Storgaard H, Mortensen B, Almdal T, Laub M, Tarnow L. At least one in three people with Type 2 diabetes mellitus referred to a diabetes centre has symptomatic obstructive sleep apnoea. Diabet Med 2014;31:1460-7.

9. Lopez PP, Stefan B, Schulman CI, Byers PM. Prevalence of sleep apnea in morbidly obese patients who presented for weight loss surgery evaluation: more evidence for routine screening for obstructive sleep apnea before weight loss surgery. Am Surg 2008;74: 834-8.

10. Netzer NC, Hoegel JJ, Loube D, et al. Prevalence of symptoms and risk of sleep apnea in primary care. Chest 2003;124:1406-14.

11. Monahan K, Redline S. Role of obstructive sleep apnea in cardiovascular disease. Curr Opin Cardiol 2011;26:541-7.

12. Eiseman NA, Westover MB, Ellenbogen JM, Bianchi MT. The impact of body posture and sleep stages on sleep apnea severity in adults. J Clin Sleep Med 2012;8:655-66A.

13. Shah P, Gurubhagavatula I. Portable monitoring: practical aspects and case examples. Sleep Med Clin 2011;6:355-66.

14. Flemons WW, Douglas NJ, Kuna ST, Rodenstein DO, Wheatley J. Access to diagnosis and treatment of patients with suspected sleep apnea. Am J Respir Crit Care Med 2004;169:668-72.

15. Masa Jimenez JF, Barbe Illa F, Capote Gil F, et al. [Resources and delays in the diagnosis of sleep apnea-hypopnea syndrome]. Arch Bronconeumol 2007;43:188-98.

16. El Shayeb M, Topfer LA, Stafinski T, Pawluk L, Menon D. Diagnostic accuracy of level 3 portable sleep tests versus level 1 polysomnography for sleepdisordered breathing: a systematic review and metaanalysis. CMAJ 2014;186:E25-51.

17. Rosen CL, Auckley D, Benca R, et al. A multisite randomized trial of portable sleep studies and positive airway pressure autotitration versus laboratorybased polysomnography for the diagnosis and treatment of obstructive sleep apnea: the HomePAP study. Sleep 2012;35:757-67.

18. Mulgrew AT, Fox N, Ayas NT, Ryan CF. Diagnosis and initial management of obstructive sleep apnea without polysomnography: a randomized validation study. Ann Intern Med 2007;146:157-66.

19. Ghegan MD, Angelos PC, Stonebraker AC, Gillespie MB. Laboratory versus portable sleep studies: a meta-analysis. Laryngoscope 2006;116:859-64.

20. Kuna ST, Gurubhagavatula I, Maislin G, et al. Noninferiority of functional outcome in ambulatory management of obstructive sleep apnea. Am J Respir Crit Care Med 2011;183:1238-44.

21. Berry RB, Hill G, Thompson L, McLaurin V. Portable monitoring and autotitration versus polysomnography for the diagnosis and treatment of sleep apnea. Sleep 2008;31:1423-31. 
22. Garg N, Rolle AJ, Lee TA, Prasad B. Home-based diagnosis of obstructive sleep apnea in an urban population. J Clin Sleep Med 2014;10:879-85.

23. Berry RB, Budhiraja R, Gottlieb DJ, et al. Rules for scoring respiratory events in sleep: update of the 2007 AASM Manual for the Scoring of Sleep and Associated Events. Deliberations of the Sleep Apnea Definitions Task Force of the American Academy of Sleep Medicine. J Clin Sleep Med 2012;8:597-619.

24. Tan HL, Gozal D, Ramirez HM, Bandla HP, Kheirandish-Gozal L. Overnight polysomnography versus respiratory polygraphy in the diagnosis of pediatric obstructive sleep apnea. Sleep 2014;37:255-60.

25. Guilleminault C, Hagen CC, Huynh NT. Comparison of hypopnea definitions in lean patients with known obstructive sleep apnea hypopnea syndrome (OSAHS). Sleep Breath 2009;13:341-7.

26. Collop NA, Anderson WM, Boehlecke B, et al. Clinical guidelines for the use of unattended portable monitors in the diagnosis of obstructive sleep apnea in adult patients. Portable Monitoring Task Force of the American Academy of Sleep Medicine. J Clin Sleep Med 2007;3:737-47.

27. Collop N. Home sleep testing: appropriate screening is the key. Sleep 2012;35:1445-6.

28. Nerfeldt P, Aoki F, Friberg D. Polygraphy vs. polysomnography: missing osas in symptomatic snorers-a reminder for clinicians. Sleep Breath 2014;18: 297-303.

29. Mokhlesi B, Tulaimat A, Faibussowitsch I, Wang Y, Evans AT. Obesity hypoventilation syndrome: prevalence and predictors in patients with obstructive sleep apnea. Sleep Breath 2007;11:117-24.

30. Laaban JP, Chailleux E. Daytime hypercapnia in adult patients with obstructive sleep apnea syndrome in France, before initiating nocturnal nasal continuous positive airway pressure therapy. Chest 2005; 127:710-5.

31. Resta O, Foschino-Barbaro MP, Bonfitto P, et al. Prevalence and mechanisms of diurnal hypercapnia in a sample of morbidly obese subjects with obstructive sleep apnoea. Respir Med 2000;94:240-6.
32. Berry RB. Uses and limitations of portable monitoring for diagnosis and management of obstructive sleep apnea. Sleep Med Clin 2011;6:309-33.

33. Hesselbacher S, Mattewal A, Hirshkowitz M, Sharafkhaneh A. Classification, technical specifications, and types of home sleep testing devices for sleep-disordered breathing. Sleep Med Clin 2011;6: 261-82.

34. Yuceege M, Firat H, Demir A, Ardic S. Reliability of the Watch-PAT 200 in detecting sleep apnea in highway bus drivers. J Clin Sleep Med 2013;9:339_ 44.

35. Pittman SD, Pillar G, Berry RB, Malhotra A, MacDonald MM, White DP. Follow-up assessment of CPAP efficacy in patients with obstructive sleep apnea using an ambulatory device based on peripheral arterial tonometry. Sleep Breath 2006;10:123-31.

36. Yalamanchali S, Farajian V, Hamilton C, Pott TR, Samuelson CG, Friedman M. Diagnosis of obstructive sleep apnea by peripheral arterial tonometry: meta-analysis. JAMA Otolaryngol Head Neck Surg 2013;139:1343-50.

37. Hedner J, White DP, Malhotra A, et al. Sleep staging based on autonomic signals: a multi-center validation study. J Clin Sleep Med 2011;7:301-6.

38. Wittine LM, Olson EJ, Morgenthaler TI. Effect of recording duration on the diagnostic accuracy of out-of-center sleep testing for obstructive sleep apnea. Sleep 2014;37:969-75.

39. Masa JF, Corral J, Pereira R, et al. Effectiveness of sequential automatic-manual home respiratory polygraphy scoring. Eur Respir J 2013;41:879-87.

40. Aurora RN, Swartz R, Punjabi NM. Misclassification of obstructive sleep apnea severity with automated scoring of home sleep recordings. Chest 2014; 147:719-27.

41. Park DY, Kim HJ, Kim CH, et al. Reliability and validity testing of automated scoring in obstructive sleep apnea diagnosis with the Embletta X100. Laryngoscope 2015;125:493-7.

42. Nigro CA, Dibur E, Aimaretti S, Gonzalez S, Rhodius E. Comparison of the automatic analysis versus the manual scoring from ApneaLink device for the diagnosis of obstructive sleep apnoea syndrome. Sleep Breath 2011;15:679-86. 